\title{
Research on the Security Enhanced Smart Hardware Assisted Regression Analysis and Health Monitoring Technique based Expanded Training Exercise Effect Evaluation Model
}

\author{
Kejin Shi \\ Huazhong Agricultural University, Wuhan, Hubei Province, China, 430070
}

\begin{abstract}
With the rapid development of the Internet of things based smart hardware technology and computer engineering, combination of computer techniques with general expanded training exercise is the irreversible trend. In this paper, we propose the security enhanced smart hardware assisted regression analysis and the health monitoring technique based expanded training exercise effect evaluation model. Our research is composed of the following parts. (1) We review state-of-the-art smart hardware device organization and security enhancement countermeasures to serve as the foundation for the further modelling. (2) We propose novel mathematical regression analysis paradigm and optimized prediction model based on the neural network theory and convex optimization to assisting the later evaluation work. (3) We propose the new health monitoring device with the enhanced security to real-time monitor the movement and physical data. (4) We compare the proposed algorithm with the other popular adopted approaches and conduct comparison experiment. The experimental result proves that our model gets the higher prediction precision which enhance the traditional precision from $87 \%$ to $96.5 \%$ and the robustness is strengthened as well. In the future, we will combine more mathematical model to modify the current model to achieve the better performance.
\end{abstract}

Keywords: Security Enhancement; Smart Hardware; Regression Analysis; Expanded Training; Exercise Effect; Evaluation Model; Health Monitoring System .

\section{Introduction}

Intelligent wearable device is a kind of media that can wear on your body or close to pass information and can send and computing devices, it can take the advantage of the information such as sensors, RFID, global positioning system sensing devices, mobile Internet access, to achieve the exchange of the information anytime and anywhere people and things. Health, information intelligent wearable devices are divided into life. The core techniques contained in the smart devices are categorized as the following aspects. (1) Data computing technology. It is limited by the device itself volume requirements, comfort and the wearable devices are relatively limited equipment and the personal computer are relatively limited in terms of the hardware resources, such as creating its high specificity in terms of function, it is difficult to further expand and extend. If rely on wearable device itself, its function and the application scenario could be very limited. Thanks to wireless communication technology, cloud platform technology and the development of the technology of data, the wearable devices combined with cloud computing and big data as the wearable devices thus becomes a wide prospect in application [1-3]. (2) Interactive technologies. With depth of optical sensor data acquisition, and then realize the human-computer interaction based on body feeling, is a research hotspot in the field of human-computer interaction in recent years. In addition to posture and gesture recognition based on infrared or video equipment technology, using acceleration sensor and the behavior of the micro sensor equipment such as mechanical 
gyroscope recognition is widely used in mobile terminals and smart TV. The corresponding tracking algorithms could be reached in the articles [4-9]. Due to the expression of language can carry almost all human intent, if through the spoken can get computer rapid feedback and service, it will make people interactions with the computer more directly and natural. (3) Sensing technology. Sensor data is the main input of the wearable devices that is the basis of a subsequent calculation analysis, and service. Wearable devices through a variety of different types of sensors from different sources of information, such as environment, human body, storage, analysis and calculation, and support the upper application service and interactive behavior. Depth sensing technology for wearable devices provide a much richer set of input information, application more is the depth of the sensing technology based on 3D technology. At present commonly used camera collection are flat 2D information, to collect 3D information usually requires additional depth of field cameras, namely the scene images were obtained through the two cameras at the same time to calculate the same effect to the human eye to collect information [10-15].

Wearable health monitoring system is the physiological information detection and wireless communication and fusion of wearable technology, as a result, because of its advantages of the small volume, light weight, easy to wear, have been in the field of military and civilian two attach great importance to the scientific research personnel, and achieved certain results. Physiological information researchers will test equipment designed to vest, rings, watches, chest, participants wear this kind of equipment, with cases often activities in does not affect the detection of human physiological information. By fusion of the posture and physiological information, the remote monitoring center of the medical staff can understand how of these physiological information are the subjects in the environment and the activities of the state acquisition, facilitate the staff analysis subjects of safety and health.

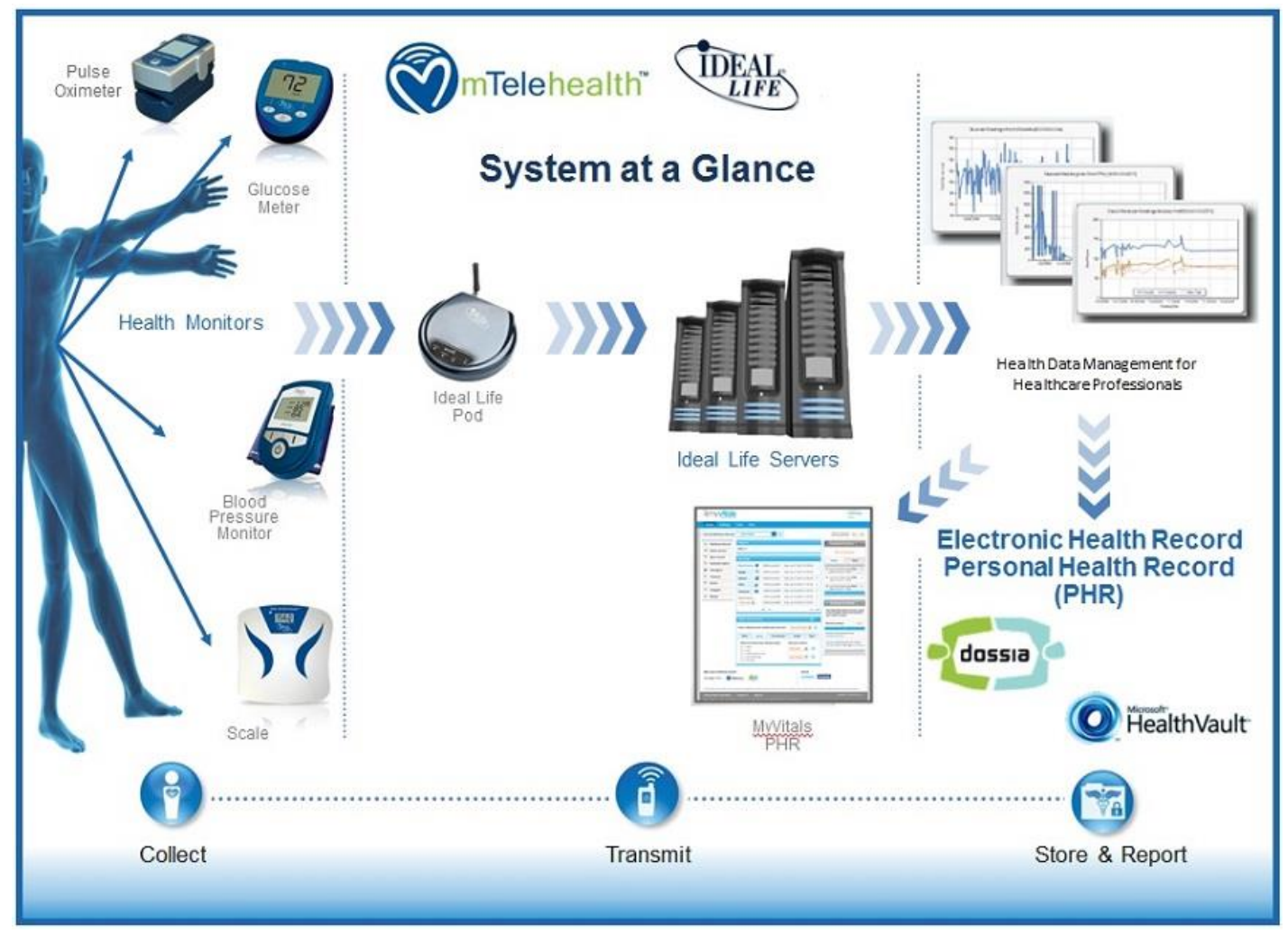

Figure 1. The Sample Healthcare Monitoring System 
As demonstrated in the figure one, we explain the sample healthcare monitoring system. Remote health management based on the technology of Internet of things will make use of the wireless sensor network as the Internet provides the information highway, for patients with all kinds of physiological parameters real-time data read, information exchange, remote control and other features. Internet of things is to point to by all kinds of information sensing device, no need to monitor the real-time acquisition, connected, interactives information need to wait for all sorts of objects or process, formed by the combination of a huge network with Internet. Its purpose is to realize the content and content, objects and people as all of the items and the network connection, easy to identify, manage and the control. Expand training follows the experiential learning model of pedagogy theory. The construction of "experiential learning circles" marks formal put forward experiential learning theory. This theory is that effective learning from experience. The experience obtained in the process of feeling, then the harvest of knowledge sharing in the team as the final agreement [16-17]. Time-series forecasting currently has become a hot spot in the field of data mining research but the characteristics of nonlinear chaotic time-series data also makes the mining of it become the difficult problem. More of direct prediction method in the aspect of modeling, artificial intelligence, at the same time, for the various influence factors in the prediction problem of the complex and nonlinear relationship between fitting ability is stronger, the prediction accuracy and the efficiency. The network training of these methods is the mostly based on the global error change network weights this principle and it is difficult to control the volatility strong prediction problem training precision. The information stored by the training after the network weight more reflects the larger sample size, flat piece of data, and on the sample data of the smaller peak data rule is difficult to effectively reflect the problem.

In this paper, we theoretically and numerically research on the topic of security enhanced smart hardware assisted regression analysis and health monitoring technique based expanded training exercise effect evaluation model. The manuscript is organized as the follows. In the second section, we discuss the state-of-the-art smart hardware devices' organization and the security enhancement countermeasures. In the third section, we propose the novel regression analysis paradigm to serve as the foundation for the further research. In the fourth section, we list the modern health monitoring technique. In fifth section, we propose optimized expanded training exercise effect evaluation model with the neural network theory and related technique as the implementation. In the last two sections, we simulation the proposed model compared with the others and conclude the paper with the future research plan.

\section{The Modern Smart Hardware Devices and Enhancement}

\subsection{The State-of-the-art Smart Hardware Devices}

Virtualization technology is a kind of effective means to improve information security, for the mobile intelligent device virtualization has become a hot spot in the field of the current operating system. On how to improve the information security and reduce two virtualization overhead lines through virtualization technology, mobile intelligent device can run multiple operating systems like server instance, mutual isolation between instance, improved the core security information. However, different from the server, intelligent equipment performance is relatively low, the introduction of virtualization technology at the same time also brought additional performance overhead, and the cost is not to be ignored. If under the condition of the virtualization, intelligent device can run multiple operating system instances and isolated, then both enhance the information security, and do not produce the additional performance overhead that can achieve the 
result of kill two birds with one stone. The current smart device could be categorized as the following categories.

$>$ Based on the exercise of the application of the wearable equipment. The daily fitness wearable device is mainly pay attention to the people through sports to strengthen body health, they can real time record their daily exercise is taken, energy, food and sleep, thus effectively and reasonably arrange their exercise.

$>$ Medical monitoring of the field. Current wearable equipment is mainly applied to the monitoring data of the human body can through the sensors to collect physiological data of the body. At present the function of the above mentioned products focus on the human body data monitoring and the human body data in real time to provide for the people's health has a significant meaning to life.

$>$ Wearable devices in the field of entertainment, social development is given priority to with smart glasses and the smart watches. In the field of the entertainment, social, smart glasses, smart watches the phone is equivalent to the entertainment function of migration to wearable devices, make entertainment device has the function can call and the use of mobile Internet applications.

Because the wearable computer has the characteristics above, the corresponding software system will face some special problems. Current international for wearable computer software architecture research in general is still relatively weak, some universities and the research institutions, according to the special features of wearable computer and is widely used to do a lot of preliminary exploration, put forward own solution. Similar with traditional intelligent terminal, wearable devices also need operation system, platform and the algorithm software technical support. Considering the specific application scenario for the wearable device as its own limited storage and computing power, they could have the relevant software technology have a higher dependence. In the following figure we show the well performed smart devices and the corresponding communication platform.

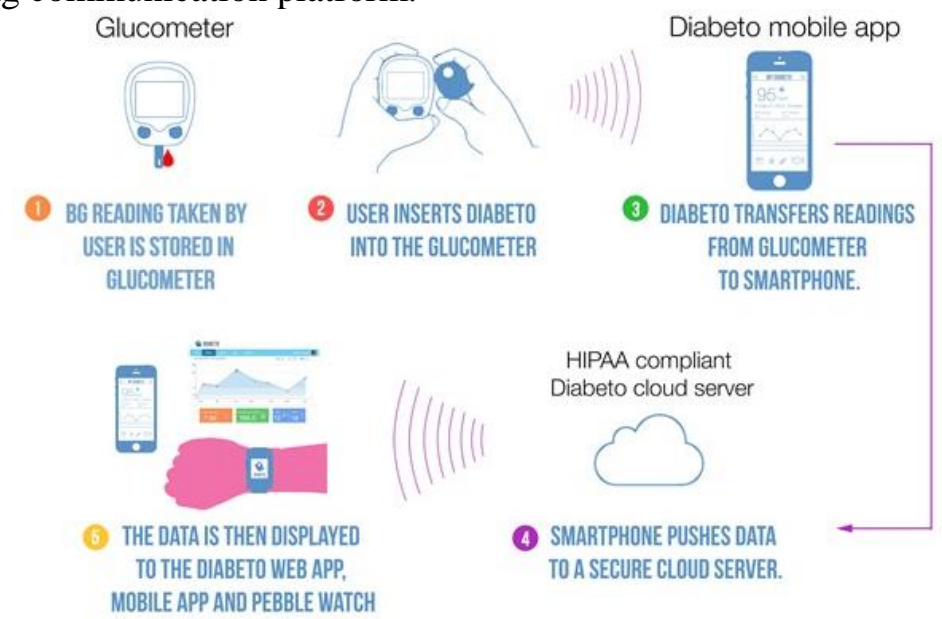

Figure 2. The Modern Smart Devices and the Communication Platform

\subsection{The Security and Organization Enhancement Trend}

Smart hardware should be reliable and the durable, every effort should be made to avoid product malfunction. In the actual design activities, due to the mechanical structure and the sensor was intelligent hardware integration module so often to have the conflict in the interior of the product, if the solution is not good will appear a series of the failures. Users in the operation of the intelligent hardware, because of its functional properties are not the familiar with, often appear wrong operation or do not conform to the specification of operation. If the product can provide the optimized fault tolerance at this moment, this 
will encourage users to try to learn the product continuously to make product can really service for the user. Product appearance and function to clear and this is similar to traditional product design requirements, all need to shape the meaning and function of corresponding directly, but the difficulty is the appearance of intelligent hardware design not only should point to the function, but also to interact with people's body language. The software control code can be modified and products intelligent one remarkable feature is the function of the product is variable with the action, it's like a car factory, the installation of the robot, called a robot is that the mechanical arm can be according to the different models for different installation sequence and the position, so as to produce different kinds of models [18-20].

The security and organization enhancement trend could be summarized as the follows. (1) High speed Internet and cloud computing. When the broadband or mobile Internet speed close to or even more than the hard disk read and write speed, through the terminal access cloud data as to be as easy to read in his hard drive. (2) Eye-tracking. Eye tracking technology is already widely used in scientific research field, especially in field of psychology. (3) Speech recognition. Speech recognition is common in some mobile operating system, software, and some sites, speech recognition technology of the smart wearable devices, can be on the input and human-computer interaction. (4) Human chip has been widely used in military and the medical fields, but at present, because of the volume and safety, human body chip technology has failed to get a wide range of applications.

\section{The Regression Analysis Paradigm}

\subsection{The Traditional Mathematical Regression Models}

In the research and application of linear regression model, normal distribution assumption, the nonlinear regression model with combination model modeling is an important research problem. Nonlinear regression model is irreplaceable, so far there are no modeling method, a nonlinear regression model usually use the thought of nonlinear linearization, the nonlinear regression model changes for linear regression model. The nonlinear linearization, however, has proven the variable substitution involves parameter is not set up [21-22].

In the practice of the application of regression model to forecast, because of the complexity of the phenomenon and the relationship between independent variable and dependent variable and the limited ability to explain the complexity of a single model, determines the application of a single model to forecast of forecast error is generated by larger, all kinds of combination model arises at the historic moment. Measure is the standard minimum of variance prediction accuracy, the many kinds of prediction method of weighted combination of prediction and get comprehensive forecast and the sum of the squares of the minimum error between the raw data are given into mathematical model are as follows.

$$
\min \sum_{i=1}^{n}\left[\beta_{1} \times x_{1}(i)+\beta_{2} \times x_{2}(i)+\ldots+\beta_{p} \times x_{p}(i)-y(i)\right]^{2}
$$

Where the $x_{p}$ represents individual measurement value and usually the established of the regression models are based on the residual obey the normal distribution assumption, however, residual tend to obey the normal distribution. Determine the non-normal distribution types parameter estimation method is used to estimate parameters. If we cannot determine the non-normal distribution types, use the parameter estimation method to estimate parameters. The corresponding restrictions of the parameters are shown as the follows. 


$$
\beta_{1}+\beta_{2}+\cdots+\beta_{p}=1
$$

The basic idea of this method is a nonlinear law shall be converted into linear regression, the original sample data for the minimum quantitative processing, until the new data under a given significance level has significant linear correlation. The formula three demonstrate the general transformation of the regression process.

$$
\begin{gathered}
\left\{\begin{array}{l}
\frac{\partial Q}{\partial \beta_{1}}=(-2) \sum_{i=1}^{n}\left(y_{i}-\beta_{1} x_{i 1}-\beta_{2} x_{i 2}-\cdots-\beta_{p} x_{i p}\right) x_{i 1}=0 \\
\frac{\partial Q}{\partial \beta_{2}}=(-2) \sum_{i=1}^{n}\left(y_{i}-\beta_{1} x_{i 1}-\beta_{2} x_{i 2}-\cdots-\beta_{p} x_{i p}\right) x_{i 2}=0 \\
\cdots \\
\frac{\partial Q}{\partial \beta_{p}}=(-2) \sum_{i=1}^{n}\left(y_{i}-\beta_{1} x_{i 1}-\beta_{2} x_{i 2}-\cdots-\beta_{p} x_{i p}\right) x_{i p}=0
\end{array}\right. \\
\Rightarrow\left\{\begin{array}{l}
\sum_{i=1}^{n} x_{i 1}^{2} \beta_{1}+\sum_{i=1}^{n} x_{i 1} x_{i 2} \beta_{2}+\cdots+\sum_{i=3}^{n} x_{i 1} x_{i p} \beta_{p}=\sum_{i=1}^{n} x_{i 1} y_{i} \\
\sum_{i=1}^{n} x_{i 2} x_{i 1} \beta_{1}+\sum_{i=1}^{n} x_{i 2}^{2} \beta_{2}+\cdots+\sum_{i=3}^{n} x_{i 2} x_{i p} \beta_{p}=\sum_{i=1}^{n} x_{i 2} y_{i} \\
\vdots \\
\sum_{i=1}^{n} x_{i p} x_{i 1} \beta_{1}+\sum_{i=1}^{n} x_{i p} x_{i 2} \beta_{2}+\cdots+\sum_{i=3}^{n} x_{i p}^{2} \beta_{p}=\sum_{i=1}^{n} x_{i p} y_{i}
\end{array}\right.
\end{gathered}
$$

Using the random variables and the distribution, the non-normal distribution into normal distribution, it was proposed based on the normal distribution and the model of modeling for non-normal distribution hypothesis provides method. From model significance perspective, multiple linear regressions is the study of causal relationship between the dependent variable and independent variables, and the comprehensive prediction model and the selection of the typical model method, there is also a causal relationship between typical predictive results of the prediction method is the influencing factors of comprehensive prediction results.

\subsection{The Novel Regression Analysis Paradigm}

In the case conditions permit, inspection regression prediction model of new data sample content to build regression prediction model of data samples of content. Otherwise, regression forecasting model for normal and abnormal group, or a control and the prediction of cases, abnormal group or control group with appropriate sample cases must be considered [23].

But because of the influence of parameter estimation is not accurate, the linear regression model and autoregressive conditional heteroscedastic model has some limitations in practical application and because heteroscedasticity nonparametric regression model is not affected by the influence of the parameter hypothesis as it can accurately describe the observed data with complex structure. Change point to mean and variance of heteroscedasticity nonparametric regression model to study the changing point and in the process of the generation of data as is affected by emergencies, its mean and variance can get the change point. The next sampling period, the completion of data collection, sensor nodes to error between the predicted values and the real value with the error of the preset threshold value is used in the comparison.

If the prediction error is less than the threshold, the sensor node and convergence is not for data transmission between nodes. Otherwise, the sensor node will be in their own model data sequence of observations collected to insert in order to the next round of the 
update model predicts that the observed value at the same time sent to the node. In the following formulas, we illustrate the modification for enhancement.

$$
Y_{i}=m\left(X_{i}\right)+\sigma\left(X_{i}\right) \varepsilon_{i}, \quad i=1,2, \ldots, n
$$

$$
\beta_{\text {Optimized }}=\left(X^{T} X\right)^{-1} X^{T} Y
$$

Linear regression model to describe the general relationship between the variable provides a mathematical model of the structure is simple, the regression coefficients and the variance is change point problem of a large number of research. In the figure three, we illustrate sample performance of the novel regression analysis paradigm.

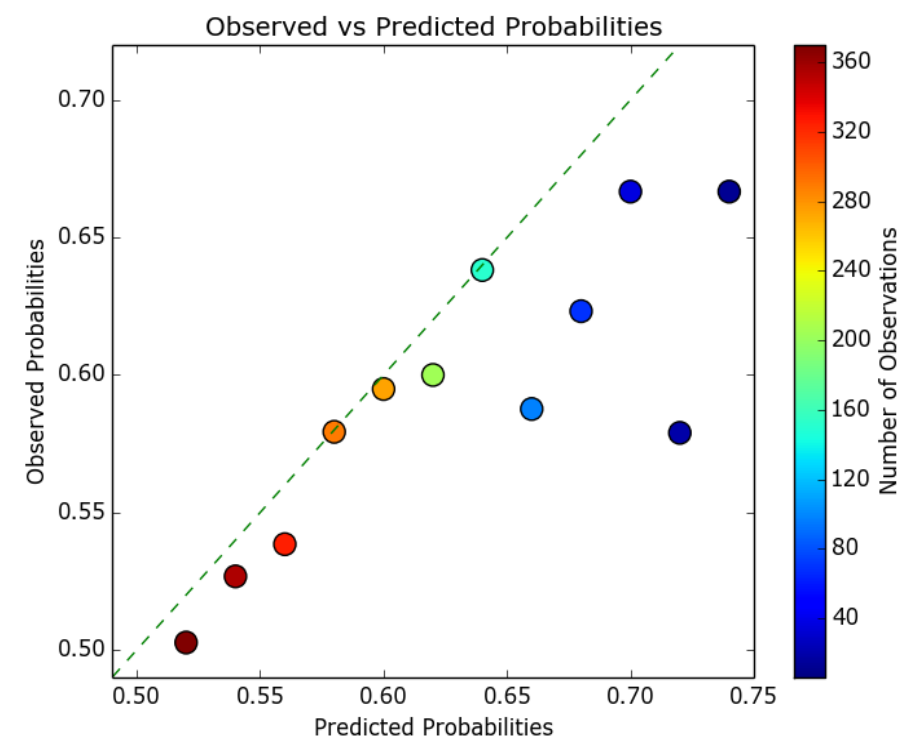

Figure 3. The Sample Performance of the Novel Regression Analysis Paradigm

\section{The Modern Health Monitoring Technique}

Remote health monitoring system based on Internet of things can mainly realize the front end acquisition human physiological parameters, using Internet and mobile communication transmission technology, ZigBee gateway, blue-tooth transmission to the guardianship use the shows at the same time, and then through the GSM to transmit the data to the remote medical monitoring on the side. The different layers for the systems are demonstrated below.

$>$ Perception layer. Perception layer is mainly used for acquisition of physical events and data in the physical world. Using intelligent sensor technology, identification and other information collection technology, the article on the basis of the information collection, receiving control information from the upper network at the same time, complete the corresponding action, and the upper network communication between done through the Internet gateway. As a gateway device, the IOT gateway can realize sensor network and communication network, and the protocol conversion between different types of sensor networks, can not only realize the wide-area interconnection which can also achieve the LAN interconnection [24].

$>$ The network layer. The network layer is equal to the person's nerve center and the brain that is responsible for the information transmission and processing system. Data management and the processing technology of the network layer are to 
achieve data-centric core technology of Internet of things. Cloud computing platform as a mass of sensory data storage, analysis platform is an important part of Internet network layer which is also the foundation of the application layer of application.

$>$ The application layer. Application layer based on sensor data to describe the specific of application. IOT application is applied to the further development of the Internet network, from the early data services as the main characteristics of the file transfer to the user as the center of the application [25-26].

Monitoring system is a kind of human physiological parameter can be a long time as the continuous and real-time acquisition human physiological parameters, has the basic function such as display, processing and preservation that can achieve a variety of application of the instrument combination which is sensor technology, computer technology, communication technology and the combination of the two physiological signal detection technology [27].

The traditional monitoring system, sensor and data processing equipment by way of cable to transmit data between a large number of the attachment affect the user's normal activities, easy to make its produce discomfort, because users of tension, not natural, the accuracy of the physiological parameters have been obtained and these factors limit the application of the physiological parameters monitoring in more fields. With the rapid development of electronic technology, especially in recent years, the short distance wireless communication technology develops very fast, some technologies have been relatively mature and the widely used, the application of the short distance of wireless communication technology to the human body physiological parameter monitoring, combined with other advanced electronic technology can realize the effect on human body small wireless monitoring system, in the case of does not affect the normal activity of charges, can undertake long-term continuous monitoring, so as to promote the development and application of biological monitoring technology.

\section{The Optimized Expanded Training Exercise Effect Evaluation Model}

\subsection{The Primary Training Exercise Effect Evaluation Model}

Data normalization methods are many, with the more normalization of linear and nonlinear normalized of the two ways. Linear normalization method using extreme value or the average operations through the linear computation formula of raw data, converts the data to a unit interval values. Nonlinear normalization method is mainly used some operations of nonlinear rows on original data, the data is converted to a certain range of data. Physical training is a continuous and dynamic process and the evaluation of training effect should also be real-time, dynamic, and approximation, fitting the training process as much as the possible. Both help trainees timely found in training is insufficient, can also help organization personnel timely analysis of the problems existing in training, make trainees and organization staff common progress and improve to achieve the enhancement purpose to the training effect [28-30].

In the field of prediction and evaluation, grey theory and Markov theory can be used for time series prediction and general evaluation of the problem. Advantage lies in the short-term forecasting of grey theory, the disadvantage is long-term forecast and volatile data columns fitting are poorer. Markov theory has the advantage of long term prediction and forecasting of random volatile data column, it can compensate for the limitations of grey theory. However, the theory of Markov prediction objects not only the requirement characteristics of Markov chain, also requires with the characteristics of a stationary processes such as mean and physical training activities most belong to change over time and present some kind of change trend of non-stationary random process. The prediction model could be summarized as below. 


$$
A=\left[\begin{array}{l}
a \\
b
\end{array}\right]=\left(B^{T} B\right)^{-1} \cdot B^{T} Y
$$

(6)

\subsection{The Neural Network based Prediction Model}

Workers of the neural network prediction method used for the nonlinear prediction effect is very good, from the perspective of the characteristics of network traffic also is nonlinear, in theory, neural network can approximate arbitrary precision any nonlinear sequence. The combination of the wavelet and neural network method, although there is a good prediction effect, but in essence or the neural network prediction, and not essentially improve the neural network training speed is slow, easy to fall into the local advantages, it is difficult to find the global minimum point defects. Another combination method is the transfer function of the hidden layer neural network instead of using wavelet function, this combination of essentially change the structure of the forecasting model, in does not affect the premise of prediction accuracy, shorten the training time of the model, improve the speed of training, to overcome the advantages of the neural network easy to fall into the local time faults, and the transfer function of the hidden layer neural network instead of using wavelet function, the algorithm is easy to implement, easy application and popularization [31-33].

From the perspective of the neural network structure, we could summarize the features as the follows. (1) From the perspective of synchronous because each neuron only after we get all the input signal can be processed so different subnet layer between the processing nodes that must synchronize or unable to handle the next layer of neurons under condition of load balancing is difficult to achieve if a process node load caused by the big data communication between nodes wait plus other costs this way it will be difficult to improve performance. (2) From the data traffic between the processing nodes in network of all neurons are connected this way of transverse subnet in parallel while nodes in terms of neurons for a processing data transmission more convenient but the connection between the properties of the resulted in each processing all need to exchange data between nodes. (3) From the perspective of load balancing on each processing node distribution on the number of neurons should be equal as far as possible contain the same number of input implied to dynamically determine hidden neurons and output neurons application means that there is no guarantee of the balance.

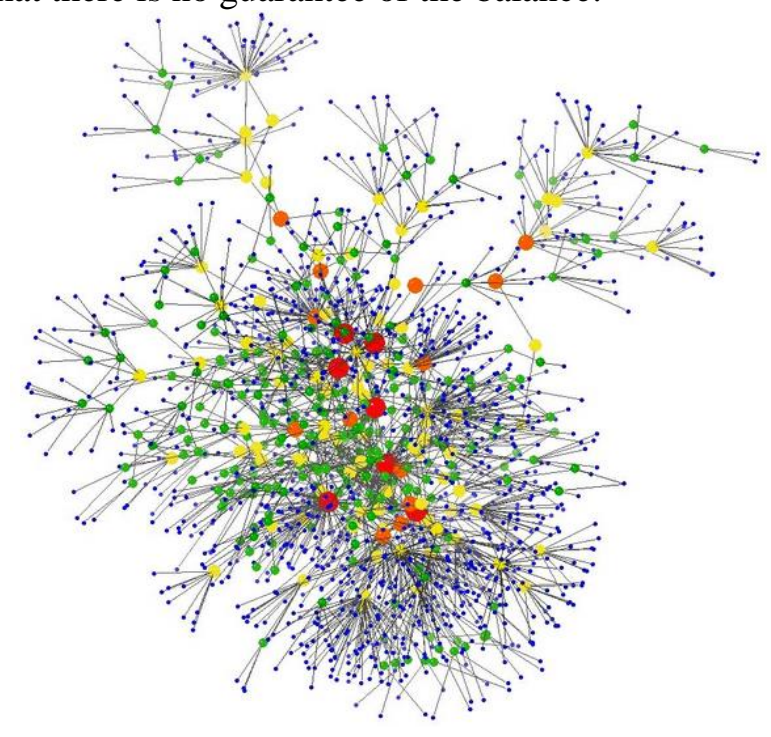

Figure 4. The Neural Network Structural Topology Ornanization 


\subsection{The Applications on the Expanded Training}

Expand training aims to emphasize and cultivate team cooperation ability and don't give up, and enterprising spirit. University is the end of most of people was a student, university sports is a bridge to the social sports, to the improvement of quality of the students has an important significance. Therefore, training is a shortcut to the efficient sports class can make the university sports teaching quality and efficiency improved. Too theoretical knowledge of the transmission of traditional teaching modes and the devotion to the teaching aim of high knowledge skills while implementing the new curriculum reform, but the reality is not satisfactory, the traditional teaching pattern, teacher supplemented students.

Due to the nature of outward bound training to meet the modern teaching idea, it quickly into campus, as long as good at using sports teachers, training must accelerate transformation of the mode of traditional teaching idea and the effect. Expand training project comes from social practice. It will be many have happened or may happen in life events and reasonable arrangement in time and space to form novel, interesting, and need the effort to complete the expansion of project. In the process of students to participate in a challenging and success or failure depends entirely on students subjective experience and psychological contradictions. It is student psychological development. Colorful nature of the outward bound training-training mode that conforms to requirement as long as we in combination with the practical situation of school take advantage, whether or not the teaching space, sports items are perfect enough which can make university sports content enrichment and diversification. To improve the students' innovative thinking and practical ability, innovation is the soul of a nation's progress, the inexhaustible driving force for prosperity of a country that is a measure of the quality of science and core technology talents and cultivating student creativity of innovative thinking is the key of the quality education. In the next section, we will simulate the proposed model to test the evaluation accuracy and effectiveness.

\section{Experiment and Simulation}

In this section, we numerically simulate the proposed model with the comparison of the standard testing paradigm. In the figure 5, we demonstrate the measurement points from large scale observation. We could conclude from the result that in most of the cases, our model gets the satisfactory prediction accuracy. In the figure 6 , we visually transfer the curve based result into the figure based demonstration, we could see from the image that our prediction model is play the robust performance when predicting the items. Finally, in the figure 7 , we show the statistical data for the testing procedures as the overall demonstration, numerically speaking our methodology enhance the traditional approaches in the large extent. 


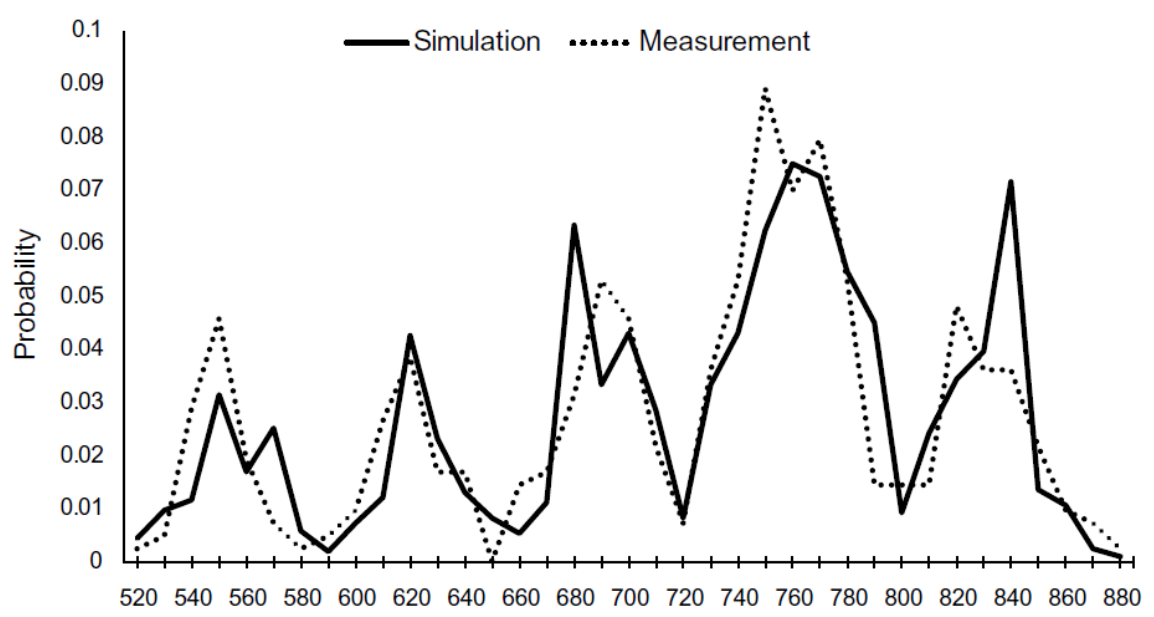

Figure 5. The Simulation Set One on the Prediction Accuracy

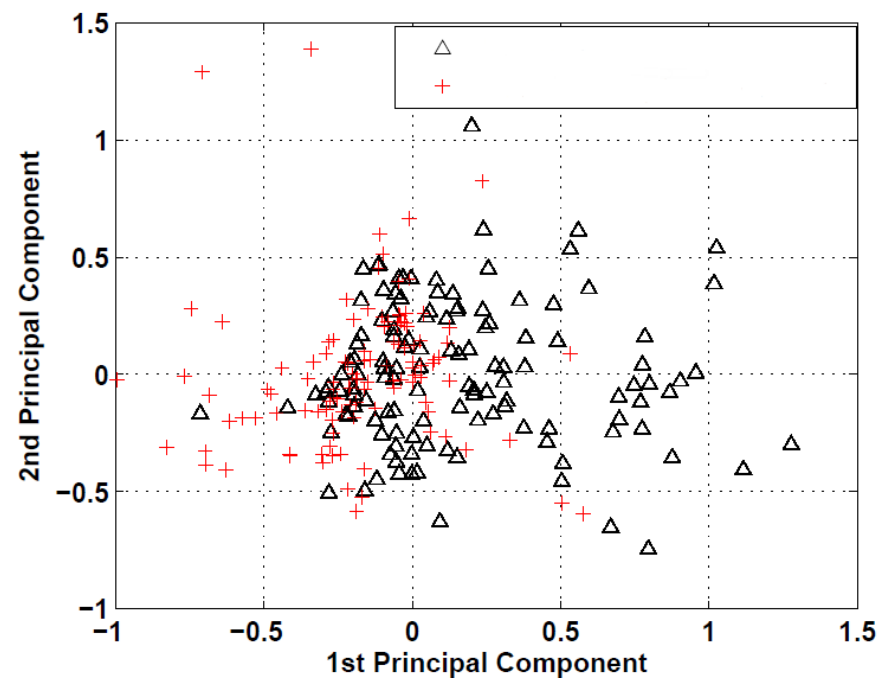

Figure 6. The Visual Illustration of the Prediction Accuracy Testing

\begin{tabular}{llll}
\hline (1) Rank & (2) Case no. & (3) Case similarity score & (4) Prediction accuracy \\
\hline 1 & 73 & 96.17991949 & 70.08053452 \\
2 & 75 & 94.71121284 & 73.94187771 \\
3 & 71 & 93.70760918 & 81.08078006 \\
4 & 67 & 89.29874029 & 83.42134734 \\
5 & 69 & 82.48743226 & 86.87114090 \\
6 & 64 & 79.48230739 & 88.21675882 \\
7 & 63 & 77.85311445 & 91.24237993 \\
8 & 70 & 74.72900797 & 69.10483470 \\
9 & 84 & 67.59786276 & 60.34882027 \\
10 & 46 & 67.56670899 & 78.84165989 \\
11 & 22 & 67.08935344 & 81.64234757 \\
12 & 76 & 65.89479349 & 55.53575086 \\
13 & 68 & 60.68032115 & 59.05701028 \\
14 & 47 & 60.37531078 & 57.33042681 \\
15 & 77 & 60.24002012 & 51.00820591 \\
16 & 81 & 57.75052784 & 96.48560101 \\
17 & 83 & 55.98643206 & 59.02520781 \\
18 & 56 & 55.79534094 & 71.04307138 \\
19 & 44 & 55.21443021 & 70.78694335 \\
20 & 20 & 54.42260658 & 83.42671891 \\
$:$ & $:$ & $:$ & $:$ \\
\hline
\end{tabular}

Figure 7. The Statistical Data and Collection of the Evaluation Performance 


\section{Final Conclusion}

In this paper, we theoretically and numerically research on the topic of security enhanced smart hardware assisted regression analysis and health monitoring technique based expanded training exercise effect evaluation model. Wearable devices are to denote the embedded in the clothing or in the form of the act the role is tasted, wear items, electronic communication equipment. Such devices can be worn comfortably and user have extended state of awareness, monitoring and improve the work efficiency. Our research analyzes and optimizes the current smart device from the following aspects. (1) Wearable equipment forms, so many different forms of products have different function and each user will wear multiple wearable devices at the same time. (2) Wearable devices mostly adopts high performance battery and low power communication module and moreover, the wearable equipment continuous working time longer than the other end. (3) Wearable devices do not need both hands to operate. As the further application, we apply the optimized regression model and prediction algorithms into training exercise effect evaluation with the corresponding health monitoring. The result as demonstrated above proves the effectiveness and robustness of the proposed model. The prediction accuracy is enhanced as the step of approximately $20 \%$. In the recent future, we will combine more complex model to optimize the current algorithm.

\section{References}

[1] Suarez-Tangil, Guillermo, Juan E. Tapiador, Pedro Peris-Lopez, and Arturo Ribagorda. "Evolution, detection and analysis of malware for smart devices." Communications Surveys \& Tutorials, IEEE 16, no. 2 (2014): 961-987.

[2] Wang, Ying, Jiaqi Zhu, Tingting Zheng, Fei Gao, and Xiang Guo. "Comparing Three Smart Device Setups for the Use of Speech Interface in Destination Search while Driving." In Transportation Research Board 94th Annual Meeting, no. 15-0469. (2015).

[3] Ren, Fuji, Mengni Chen, and Yu Gu. "WeWatch: Bi-screen video watching experience on smart devices." In Cloud Computing and Intelligence Systems (CCIS), 2014 IEEE 3rd International Conference on, pp. 265-270. IEEE, (2014).

[4] Pillai, Jagan A., and Sheryl R. Haut. "Patients with epilepsy and psychogenic non-epileptic seizures: an inpatient video-EEG monitoring study." Seizure 21, no. 1 (2012): 24-27.

[5] Tahir, Syed Fahad, and Andrea Cavallaro. "Low-cost multi-camera object matching." In Acoustics, Speech and Signal Processing (ICASSP), 2014 IEEE International Conference on, pp. 6869-6873. IEEE, (2014).

[6] Yin, Fei, Sergio A. Velastin, Tim Ellis, and Dimitrios Makris. "Learning multi-planar scene models in multi-camera videos." IET Computer Vision 9, no. 1 (2014): 25-40.

[7] Wang, Haoxiang, Ferdinand Shkjezi, and Ela Hoxha. "Distance metric learning for multi-camera people matching." In Advanced Computational Intelligence (ICACI), 2013 Sixth International Conference on, pp. 140-143. IEEE, (2013).

[8] Urakova, N. A., and A. L. Urakov. "Diagnosis of Intrauterine Brain Hypoxia Using Thermal Imaging Video Monitoring of the Fetus." Biomedical Engineering 48, no. 3 (2014): 111-115.

[9] Thompson, Donald, Christopher C. Caudill, Cristi Negrea, and Frank Loge. "Underwater Video Monitoring of Adult Fish Ladder Modifications to Improve Pacific Lamprey Passage at McNary, Ice Harbor, Little Goose, and Lower Granite Dams, 2013." (2014).

[10] Wang, Dongxiang, Xiang Ling, Hao Peng, Lin Liu, and LanLan Tao. "Efficiency and optimal performance evaluation of organic Rankine cycle for low grade waste heat power generation." Energy 50 (2013): 343-352.

[11] Sanchez, F., L. Moliner, C. Correcher, A. Gonzalez, A. Orero, M. Carles, A. Soriano et al. "Small animal PET scanner based on monolithic LYSO crystals: performance evaluation." Medical physics 39, no. 2 (2012): 643-653.

[12] Abed, Mansour, Adel Belouchrani, Mohamed Cheriet, and Boualem Boashash. "Time-frequency distributions based on compact support kernels: properties and performance evaluation." Signal Processing, IEEE Transactions on 60, no. 6 (2012): 2814-2827.

[13] Sluban, Borut, Dragan Gamberger, and Nada Lavrač. "Ensemble-based noise detection: noise ranking and visual performance evaluation." Data mining and knowledge discovery 28, no. 2 (2014): 265-303.

[14] Li, Wenyu, Chao Zhang, Xiaoyu Duan, Shucong Jia, Yu Liu, and Lin Zhang. "Performance evaluation and analysis on group mobility of mobile relay for LTE advanced system." In Vehicular Technology Conference (VTC Fall), 2012 IEEE, pp. 1-5. IEEE, (2012). 
[15] Pai, Fu-Sheng, Ru-Min Chao, Shin Hong Ko, and Tai-Sheng Lee. "Performance evaluation of parabolic prediction to maximum power point tracking for PV array." Sustainable Energy, IEEE Transactions on 2, no. 1 (2011): 60-68.

[16] Haque, Ashraf Ul, Paras Mandal, Julian Meng, and Ricardo L. Pineda. "Performance evaluation of different optimization algorithms for power demand forecasting applications in a smart grid environment." Procedia Computer Science 12 (2012): 320-325.

[17] Eghbali, Hamed, Koen Sandra, Frederik Detobel, Frederic Lynen, Kazuki Nakanishi, Pat Sandra, and Gert Desmet. "Performance evaluation of long monolithic silica capillary columns in gradient liquid chromatography using peptide mixtures." Journal of Chromatography A 1218, no. 21 (2011): 33603366.

[18] Deng, Xue, Min Zhang, and Feng Xiao. "Design and Application of Intelligent Transportation System Based on The Internet of Things." In 2014 International Conference of Logistics Engineering and Management (ICLEM). (2014).

[19] Lee, ChangYong, Fumiya Matsuno, Yoshinori Hashimoto, Hiroshi Okada, Kazuaki Sawada, and Akihiro Wakahara. "Intelligent ultraviolet sensor composed of GaN-based photodiode and N-channel metal oxide semiconductor Si-charge transfer type signal processor." Japanese Journal of Applied Physics 51, no. 4R (2012): 044101.

[20] Lan, Zhen Ping, Yu Ru Wang, Ping Li, Yun Duo Lin, Hui Min Meng, and Zhi Sen Wang. "Design of an Intelligent Help System." In Applied Mechanics and Materials, vol. 538, pp. 285-288. (2014).

[21] Kavousi-Fard, Abdollah, Haidar Samet, and Fatemeh Marzbani. "A new hybrid modified firefly algorithm and support vector regression model for accurate short term load forecasting." Expert systems with applications 41, no. 13 (2014): 6047-6056.

[22] Rust, Henning W., Andy Richling, Peter Bissolli, and Uwe Ulbrich. "Linking teleconnection patterns to European temperature-a multiple linear regression model." Meteorologische Zeitschrift (2015).

[23] Li, Mingxiang. "Moving Beyond the Linear Regression Model Advantages of the Quantile Regression Model." Journal of Management (2014): 0149206314551963.

[24] Hackmann, Gregory, Weijun Guo, Guirong Yan, Zhuoxiong Sun, Chenyang Lu, and Shirley Dyke. "Cyber-physical codesign of distributed structural health monitoring with wireless sensor networks." Parallel and Distributed Systems, IEEE Transactions on 25, no. 1 (2014): 63-72.

[25] Wu, Nan, Xiaofeng Cheng, Qize Zhong, Junwen Zhong, Wenbo Li, Bo Wang, Bin Hu, and Jun Zhou. "Cellular Polypropylene Piezoelectret for Human Body Energy Harvesting and Health Monitoring." Advanced Functional Materials 25, no. 30 (2015): 4788-4794.

[26] Loesch Vianna, Wlamir Olivares, Luiz Gonzaga de Souza Ribeiro, and Takashi Yoneyama. "Electro hydraulic servovalve health monitoring using fading extended Kalman filter." In Prognostics and Health Management (PHM), 2015 IEEE Conference on, pp. 1-6. IEEE, (2015).

[27] Costa Antunes, Paulo, João Miguel Dias, Humberto Varum, and Paulo André. "Dynamic structural health monitoring of a civil engineering structure with a POF accelerometer." Sensor Review 34, no. 1 (2014): 36-41.

[28] Moons, Karel GM, Douglas G. Altman, Johannes B. Reitsma, and Gary S. Collins. "New Guideline for the Reporting of Studies Developing, Validating, or Updating a Prediction Model." Clinical chemistry 61, no. 3 (2015): 565-566.

[29] Novianti, Putri W., Victor L. Jong, Kit CB Roes, and Marinus JC Eijkemans. "Factors affecting the accuracy of a class prediction model in gene expression data." BMC bioinformatics 16, no. 1 (2015): 199.

[30] Jansen, Marc H., Sophie E. Veldhuijzen van Zanten, Esther Sanchez Aliaga, Martijn W. Heymans, Monika Warmuth-Metz, Darren Hargrave, Erica J. van der Hoeven et al. "Survival prediction model of children with diffuse intrinsic pontine glioma based on clinical and radiological criteria." Neurooncology 17, no. 1 (2015): 160-166.

[31] Guo, Zhen-hai, Jie Wu, Hai-yan Lu, and Jian-zhou Wang. "A case study on a hybrid wind speed forecasting method using BP neural network." Knowledge-based systems 24, no. 7 (2011): 1048-1056.

[32] Liu, Qingshan, and Jun Wang. "Finite-time convergent recurrent neural network with a hard-limiting activation function for constrained optimization with piecewise-linear objective functions." Neural Networks, IEEE Transactions on 22, no. 4 (2011): 601-613.

[33] Kant, Amal, Pranmohan K. Suman, Brijesh K. Giri, Mukesh K. Tiwari, Chandranath Chatterjee, Purna C. Nayak, and Sawan Kumar. "Comparison of multi-objective evolutionary neural network, adaptive neuro-fuzzy inference system and bootstrap-based neural network for flood forecasting." Neural Computing and Applications 23, no. 1 (2013): 231-246. 
International Journal of Security and Its Applications

Vol. 10, No. 2 (2016) 